\title{
Extensive Abdominopelvic Desmoplastic Small Round Cell Tumor - Case Report and Review of the Literature
}

\author{
Adrienne Horváth11,2, Zsuzsanna Erzsébet Papp \\ 1 University of Medicine and Pharmacy, Tîrgu Mureș, Romania \\ 2 Department of Pediatrics, County Emergency Clinical Hospital, Tîrgu Mures, Romania
}

\section{CORRESPONDENCE}

Adrienne Horváth

Str. Gheorghe Marinescu nr. 38

540139 Tîrgu Mureș, Romania

Tel +40 265215551

E-mail: adigyer1@yahoo.com

\section{ARTICLE HISTORY}

Received: 24 August, 2016

Accepted: 10 September, 2016

\begin{abstract}
Desmoplastic small round cell tumor (DSRCT) is a rare malignant tumor, which affects mostly young males and has a poor prognosis. Since 1991, when it was first described as a distinct clinical entity by Gerald WL and Rosai J, some 200 cases were reported. DSRCT arises mainly from the abdominal and pelvic cavity, causes abdominal pain or discomfort, weight loss, urinary, bowel or bile obstruction due to compression. Metastases appear most frequently in the liver and lungs. Multimodal therapy is usually indicated with chemotherapy, surgery, radiotherapy and autologous stem cell transplantation. DSRCT should be differentiated from other small, blue round cell tumors, especially hematopoietic malignancies (leukemia, lymphoma), neuroblastoma, Ewing sarcoma, PNET, rhabdomyosarcoma, malignant mesothelioma, small cell carcinoma and Wilms tumors. We report the case of a patient with an extensive abdominopelvic desmoplastic small round cell tumor, with liver metastases, in an adolescent male patient, highlighting the alert deterioration of the clinical status of the patient after the biopsy, the need for a second review of the histopathological material in order to obtain a correct diagnosis, the chemoresistance of the tumor despite an apparently good clinical status, and the severe prognosis of this type of tumor.
\end{abstract}

Keywords: desmoplastic small round cell tumor, adolescence, abdominal tumor

\section{INTRODUCTION}

Desmoplastic small round cell tumor (DSRCT) is a rare malignant tumor, which affects mostly young males and has a poor prognosis. It was first described in 1991 by Gerald WL and Rosai J. ${ }^{1}$ Approximately 200 cases were described in the English literature, predominantly affecting the male gender (male:female ratio of $4: 1$ ). ${ }^{2}$ It is an undifferentiated soft tissue tumor with unclear histogenesis, which might be derived from primitive mesenchymal cells or neuroectodermal and primitive mesenchymal tissue. ${ }^{3}$ Here we report an extensive abdominopelvic desmoplastic small round cell tumor with liver metastases in an adolescent male patient, highlight the alert deterioration of the clinical status of the patient after the biopsy, the need for a second review of the histopathological material toward obtaining the 


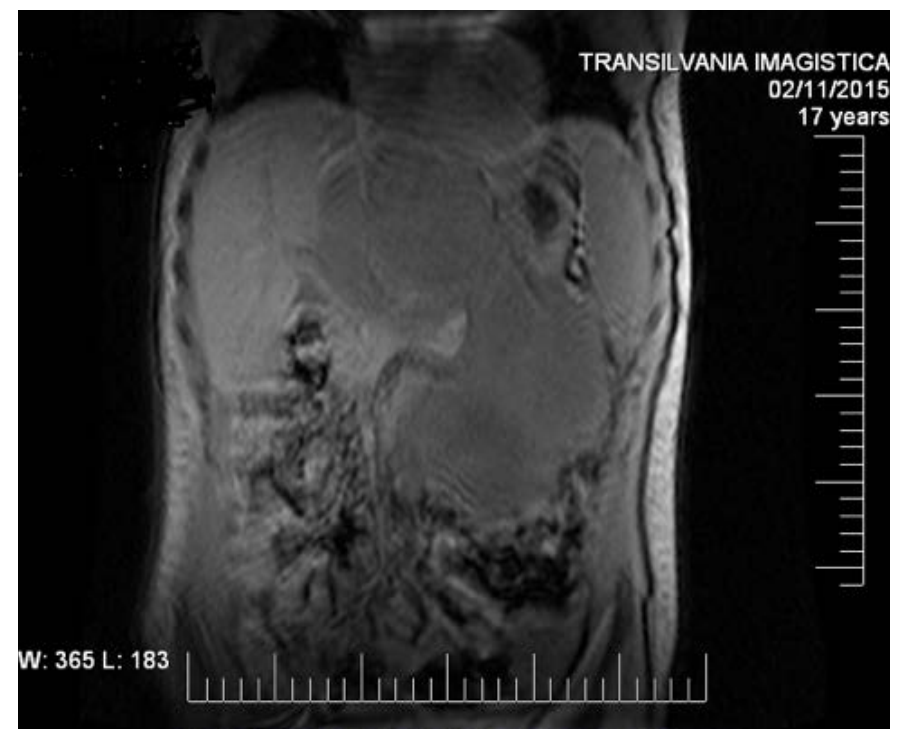

FIGURE 1. Inhomogeneous, multiple well-circumscribed tissular lesions in the retroperitoneal region which dislocate the abdominopelvic organs without invading them.

correct diagnosis, the chemoresistance of the tumor in spite of an apparently good clinical status, and the severe prognosis of this type of tumor.

\section{CASE REPORT}

The 17-year-old adolescent whose father has been on palliative care for colon adenocarcinoma, was admitted for fatigue, weight loss of $10 \mathrm{~kg}$ throughout the last 3 months, and abdominal pain in the last two weeks. On physical examination, we observed an adolescent with a body weight of $85 \mathrm{~kg}$ and height of $188 \mathrm{~cm}$, with the vital signs in the normal range, and multiple painless tumor masses of hard consistency in the abdominal cavity, mainly in the epigastrium and left abdomen, a mildly enlarged liver, of approximately $3 \mathrm{~cm}$ below the rib cage. Laboratory tests: cell blood count (CBC) and differential was in the normal range - white blood cells (WBC) $5.11 \times 10^{9} / \mathrm{L}$, haemoglobin $13.93 \mathrm{~g} / \mathrm{dl}$, haematocrit $41 \%$, platelets $169 \times 10^{9} / \mathrm{L}$, bazophils $2 \%$, segmented $54 \%$, eosinophils $2 \%$, monocytes $8 \%$, lymphocytes $34 \%$. Biochemical investigations revealed increased ASAT (107 U/L), ALAT (195 U/L) and uric acid $(8.4 \mathrm{mg} / \mathrm{dL})$ levels, higher ESR $(38 \mathrm{~mm} / \mathrm{h})$. Serology for viral infections such as hepatitis B, hepatitis C, EBV, HIV 1 and 2, were negative. Abdominal ultrasound examination revealed a wellcircumscribed, inhomogeneous hypo- and hyper echogenic tumor mass in the left lobe of the liver of $13 / 12 \mathrm{~mm}$, which compressed the pancreas. Other masses were found with the same characteristics, with a size of $86 / 98 \mathrm{~mm}$ in the rectal area and of $7 / 17 \mathrm{~mm}$ in the left abdominal flank.

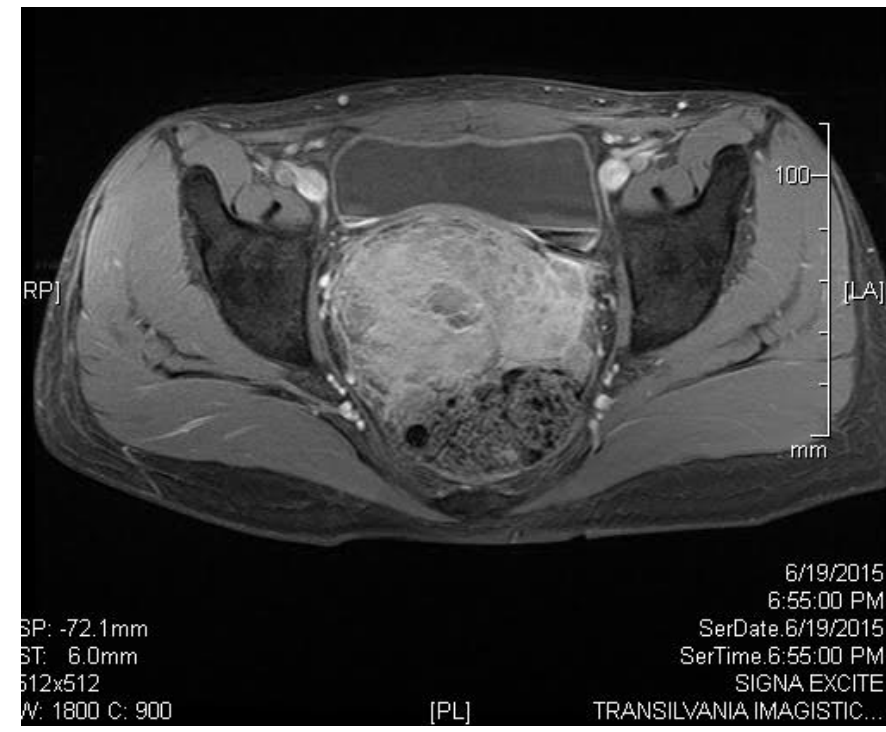

FIGURE 2. Retrovesical tumor mass with the same characterstics as in Figure 1, which compresses the urinary bladder, rectum and ileal gut.

The spleen measured $147 \mathrm{~mm}$ in the long axis. Abdominal and pelvic magnetic resonace imaging (MRI) showed multiple inhomogeneous, well-circumscribed tissular lesions in the retroperitoneal region, which dislocated the abdomino-pelvic organs and vascular structures without invading them: three hepatic subcapsular lesions of $50 / 30 / 65 \mathrm{~mm}$, $30 / 20 / 15 \mathrm{~mm}$ and $47 / 30 / 15 \mathrm{~mm}$ respectively; one lesion of $130 \mathrm{~mm}$, which compressed the left lobe of the liver and the homonymous branch of the portal vein, and dislocated the stomach; as well as multiple lesions between the rectum and the urinary bladder, the largest being $102 \mathrm{~mm}$ in size. There were three lesions in the left abdominal flank, the largest being $135 \mathrm{~mm}$ in size, which compressed the left kidney and the large curvature of the stomach. Inside the liver, there were lesions with a maximal size of $9 \mathrm{~mm}$, suggestive for secondary determinations.

Bone marrow aspiration and cerebrospinal fluid examinations were negative. Vanilmandelic acid excretion in the 24-hour urine sample was normal. An inguinal mass was excised surgically, and the histopathological examination of the tumor showed metastasis of an alveolar rhabdomyosarcoma. Histological reexamination of the sample indicated a desmoplastic small round cell tumor grade III. In the tumoral stroma, the small tumor cells with hyperchromic nuclei were organized into nests, cords and sheets, some of them showed central necrosis and cystic degeneration ( $\leq 50 \%$ of the total tumor tissue). The nests, cords and sheets were separated by an intensely vascularized desmoplastic stroma. Most of the tumor cells were small and medium-sized with a small amount of cytoplasm. There 
were regions where the tumor cells presented epithelial, muscular differentiation and necrosis or formed rosettes; here the cells were larger, with increased cytonuclear pleomorphism. Mitosis was frequent, 15-20/HPF and there were apoptotic bodies. The immunophenotype of the cells was polymorph: they expressed markers of epithelial, muscular and neural differentiation. They were cytokeratin positive, NSE, CD56, PLAT and CD99 positive. They expressed vimentin, desmin but not myogenin, which is compulsory in a rhabdomyosarcoma. The WT1 protein marked the nuclei of tumor cells, but was also expressed in the perinuclear cytoplasm. The morphology and variable immunophenotype of the tumor cells (but without expressing myogenin), pointed to DSRCT grade III. The diagnosis was also supported by the localization of the tumor, the age and gender of the patient.

The condition of the patient deteriorated in the two weeks' time between the biopsy and histological diagnosis, he became cachectic (66 kg), jaundiced with direct hyperbilirubinemia (total bilirubin $15 \mathrm{mg} / \mathrm{dL}$, direct bilirubin $10.5 \mathrm{mg} / \mathrm{dL}$ ), and moderate ascites. After insertion of a Hickmann-type central venous line, the patient underwent chemotherapy according to the CWS 2009 protocol. He received four cycles of I2 VAd (Ifosfamide, Uromitexan, Vincristin, Doxorubicin), and 2 cycles of I2VA (Ifosfamide, Mesna, Vincristin, Actinomycin-D) course in an alternating mode. Complications appeared after the first and fourth courses of chemotherapy. After the first course of I2VAd, he presented grade 4 hematological and gastrointestinal toxicity (ascites, hypoproteinemic edema, elevated liver enzymes and predominantly direct hyperbilirubinemia), febrile neutropenia, signs of intracranial hypertension with headache and dizziness. These symptoms were improved after supportive treatment with wide spectrum antibiotics, antifungal therapy, intravenous immunoglobulins, diuretics, packed red cells and platelet transfusions, granulocyte/ monocyte colony stimulating growth factors, and hepatoprotective agents. After the fourth course of chemotherapy he complained of painful passage of stools, fresh blood in the stool, on physical examination an external hemorrhoid was observed. Per oral lactulose sirup and local treatment had alleviated the symptoms related to the hemorrhoid.

Imagistic reassessment performed after three courses of chemotherapy, by thoracic computed tomography showed no lung metastases, but the abdominopelvic MRI showed no improvement compared to the first evaluation. The patient and his family expressed their option to continue with chemotherapy and postpone the surgical intervention. After six courses of chemotherapy, abdominal MRI showed unfavorable response, with multiple liver lesions up to $83 \mathrm{~mm}$, which led to compression and invasion of the hilum, associated with intrahepatic biliary tract dilations, and multiple abdominal tumor masses, mostly retroperitoneal, of up to $129 \mathrm{~mm}$ diameter. Debulking (cytoreduction) surgery was attempted in October 2015, in a relatively good clinical status and acceptable laboratory parameters including hemostasis, but the patient died during a hemorrhagic complication, the day after the surgery, in spite of intense supportive therapy with blood products in the intensive care unit. The survival time was eight months.

\section{DISCUSSION}

In $90 \%$ of cases, this highly aggressive sarcomatous tumor arises in the abdominal or pelvic cavity, and spreads along peritoneum- and mesothelial-lined surfaces, but other localizations may involve the paratesticular region, the pleural serosa, the brain, bones, ovaries, the parotid gland, the lung, stomach, pancreas and the paranasal sinuses. ${ }^{3-7}$ Metastases appear especially in the liver, with or without ascites, in the lungs, lymph nodes (groin, neck, mediastinum, abdomen) and bones. ${ }^{3,4}$ This rapidly growing tumor presents with abdominal discomfort, distention or pain, weight loss, hepatomegaly with ascites, bowel, bile or urinary tract obstruction due to compression, and other rare signs and symptoms according to its localization. On physical examination, the abdominal mass has a hard texture and low degree of mobility. ${ }^{4}$ Imagistic evaluation is important; however, it cannot establish the diagnosis. The ultrasound cystic-solid mass aspect is the result of the intratumoral necrosis and hemorrhage. The CT examination reveals intraperitoneal single or multiple soft tissue masses without an apparent organ of origin. Usually, the primary tumor occupies the retrovesical or retrouterine space. Calcification may be seen in the primary tumor and in the metastases. CT scan is also used for detecting visceral (lung/liver) metastases. ${ }^{8}$ MRI delineates the extent of the disease, so it can be helpful in planning the surgical intervention. DSRCT shows an intense uptake of the fluorodeoxyglucose tracer (FDG) on PET/CT; this may have a role in staging and follow-up 5 . On gross examination, the tumor is large with a bosselated surface, with gray-tan cut-surfaces and areas of necrosis. Microscopically, it consists of nests of small round blue cells separated by abundant desmoplastic stroma. The tumor cells are small/medium sized, with oval or round hyperchromatic nuclei and inconspicuous nucleoli. The cytoplasm is scanty and nonvacuolated. The nuclear/ cytoplasmic ratio is high. Immunohistochemical features of a DSRCT show a divergent differentiation with immunoreactivity for epithelial (keratin, epithelial membrane antigen), mesenchymal (vimentin, desmin, smooth-muscle ac- 
tin, muscle specific actin), neural (neuron specific enolase, synaptophysin, chromogranin, CD56), and miscellaneous (CD15, WT1, Ca125) markers. ${ }^{3,9,10}$ Cytogenetics of tumor cells show reciprocal chromosome translocation, $\mathrm{t}(911 ; 22)$ (p13;q12), a fusion of the N-terminal region of the Ewing sarcoma gene (EWS) located at 22q12, and the C-terminus of Wilms tumor gene (WT1) located at $11 \mathrm{p} 13$ to produce a tumor-specific fusion protein, EWS-WT1.3,11 DSRCT should be distinguished from other small, blue round cell tumors, especially hematopoietic malignancies (leukemia, lymphoma), neuroblastoma, Ewing sarcoma, PNET, rhabdomyosarcoma, malignant mesothelioma, small cell carcinoma and Wilms tumor. ${ }^{3,9} \mathrm{~A}$ widely accepted staging system for DSRCT would allow an objective comparison of different treatment methods.12,13 Prognosis is poor, especially in abdominal and pelvic localisations, with survival rates at three years of $29 \%$ and at five years of only $18 \% .{ }^{8}$ Poor prognostic factors are: metastatic disease, tumor volume exceeding $100 \mathrm{ml}$, axial site involvement, lack of response to therapy and relapse. Median survival rate is longer with autologous stem cell transplantation, even in patients with residual or persistent disease. ${ }^{14}$ There is no standardized treatment for DSRCT, but multimodal therapy is usually indicated, with chemotherapy, surgery, radiotherapy and autologous stem cell transplantation. Debulking surgery with removal of at least $90 \%$ of the tumor mass is the mainstay of the therapy, which can prolong survival. In a study performed by Lal et al., the 3-year survival rate was $58 \%$ in patients treated with debulking surgery along with chemo- and radiotherapy, compared to no survivors beyond three years in the cohort without resection. ${ }^{15}$ Wong $\mathrm{H}$ et al. described a group of 41 patients from the United Kingdom, with a median overall survival of 47 months for those with non-metastatic abdominal tumors who underwent debulking surgery, and only 16 months for those without surgery. ${ }^{16}$ Ewing sarcoma-based polychemotherapy is a frequently used therapeutic method. ${ }^{17}$ Whole abdominopelvic radiotherapy with 30 Gy and an increased radiation dose towards the remaining tumors prolonged survival, but caused significant immediate gastrointestinal and hematological side effects and late toxicity consisting of small bowel obstruction and ureteral stenosis. Relapses occurred in the radiation field. In clinical trials, new treatment modalities are being experimented with, such as postoperative abdominopelvic Intensity Modulated Radiation Therapy (IMRT), hyperthermic intraperitoneal chemotherapy (HIPEC), or treatment of hepatic metastases with Yttrium microsphere liver embolization. ${ }^{8}$

We reported an extensive abdominal desmoplastic small round cell tumor with liver metastases in an adolescent male patient, highlighting the alert deterioration of the clinical status of the patient after the biopsy, the need of second review of the histopathological material to obtain the correct diagnosis, the chemoresistance of the tumor despite an apparently good clinical status, and the severe prognosis of DSRCT.

\section{DISCLOSURE}

The authors did not receive grants, contracts or any other form of financial support for this case report.

\section{REFERENCES}

1. Gerald WL, Miller HK, Battifora H, Miettinen M, Silva EG, Rosai J. Intraabdominal desmoplastic small round-cell tumor: report of 19 cases of a distinctive type of high-grade polyphenotypic malignancy affecting young individuals. Am J Surg Pathol. 1991;15:499-513.

2. Gerald WL, Ladanyi M, De Alava E, et al. Clinical, pathologic and molecular spectrum of tumors associated with $\mathrm{t}(11 ; 22)(\mathrm{p} 13 ; \mathrm{q} 12)$ : desmoplastic smal round-cell tumor and its variants. J Clin Oncol. 1998;16(9):3028-3036.

3. Chang F. Desmoplastic Small Round Cell Tumors: Cytologic, Histologic, and immunohistochemical Features. Arch Pathol Lab Med. 2006;130:728732

4. Xiang L, Jing Y, Shibao F, Xiaoming X, Jie Z. Desmoplastic small round cell tumor: a case report and review of the literature. World Journal of Surgical Oncology. 2014;12:9.

5. Kis B, O'Regan KN, Agoston A, Javery O, Jaganathan J, Ramaiya $\mathrm{NH}$. Imaging of desmoplastic small round cell tumours in adults. $\mathrm{Br} \mathrm{J}$ Radiol. 2012;85(1010):187-192

6. Zhang G, Zhu Y, Gan H, Ye D. Testicular desmoplastic small round cel tumor: a case report and review of the literature. World J Surg Oncol. 2014;12:227

7. Jian Z, Shaohong H, Wenzhao Z, Lija G. Misdiagnosed desmoplastic round cell tumor of the pleura: Case report and literature review. J Formos Med Assoc. 2014;113(1):60-61.

8. Dufresne A, Cassier P, Couraud L, et al. Desmoplastic Small Round Cell Tumor: Current Management and Recent Findings. Clin Sarcoma Res. 2013;3:14

9. Parham DM. Small Round Cell Tumors. In: Miettinen M, editor. Modern Soft Tissue Pathology: Tumors and Non-neoplastic conditions, New York Cambridge University Press; 2010. p 896-929.

10. Klijanienko J, Colin P, Couturier J, et al. Fine-Needle Aspiration in Desmoplastic Small Round Cell Tumor. A report of 10 New Tumors in 8 Patients With Clinicopathological and Molecular Correlations With Review of the Literature. Cancer Cytopathol. 2014;122(5):386-393.

11. Miwa S, Kitamura S, Shirai T, et al. Desmoplastic small round cell tumour successfully treated with caffeine-assisted chemotherapy: a case report and review of the literature. Anticancer Res. 2010;30(9):3769-3774.

12. Hayes-Jordan A, Green H, Fitzgerald N, Xiao L, Anderson P. Novel treatment for desmoplastic small round cell tumor: hyperthermic intraperitoneal perfusion. J Pediatr Surg. 2010;45(5):1000-1006.

13. Harmon RL, Sugarbaker $\mathrm{PH}$. Prognostic indicators in peritoneal carcinomatosis from gastrointestinal cancer. Int Semin Surg Oncol. 2005;2:3.

14. Cook R, Wang Z, Arora M, et al. Clinical outcomes of patients with desmoplastic small round cell tumor of the peritoneum undergoing autologous HCT: a CIBMTR retrospective analysis. Bone Marrow Transplant. 2012;4757(10):1455-1458.

15. Lal DR, Su WT, Wolden SL, Loh KC, Modak SL. Results of multimodal treatment for desmoplastic small round cell tumors. J Pediatr Surg. 2005;40(1):251-255

16. Wong $\mathrm{HH}$, Hatcher $\mathrm{HM}$, Benson $\mathrm{C}$, et al. Desmoplastic small round cell tumour: characteristics and prognostic factors of 41 patients and review of the literature. Clin Sarcoma Res. 2013;3(1):14

17. Watanabe T, Miyamoto S, Kitagori K, et al. A case of long-term survival of metastatic desmoplastic small round cell tumor treated with multimodal therapy. Oncol Lett. 2012;3(1):30-34. 